REVIEW

\title{
Hyaluronic acid gel fillers in the management of facial aging
}

\author{
Fredric S Brandt' \\ Alex Cazzaniga ${ }^{2}$ \\ 'Private Practice in Coral Gables, \\ Florida, USA and Manhattan, NY, USA, \\ and Dermatology Research Institute, \\ Coral Gables, FL, USA; ${ }^{2}$ Dermatology \\ Research Institute, Coral Gables, \\ Florida, USA
}

\begin{abstract}
Time affects facial aging by producing cellular and anatomical changes resulting in the consequential loss of soft tissue volume. With the advent of new technologies, the physician has the opportunity of addressing these changes with the utilization of dermal fillers. Hyaluronic acid (HA) dermal fillers are the most popular, non-permanent injectable materials available to physicians today for the correction of soft tissue defects of the face. This material provides an effective, non invasive, non surgical alternative for correction of the contour defects of the face due to its enormous ability to bind water and easiness of implantation. HA dermal fillers are safe and effective. The baby-boomer generation, and their desire of turning back the clock while enjoying an active lifestyle, has expanded the popularity of these fillers. In the US, there are currently eight HA dermal fillers approved for commercialization by the Food and Drug Administration (FDA). This article reviews the innate properties of FDA-approved HA fillers and provides an insight on future HA products and their utilization for the management of the aging face.
\end{abstract}

Keywords: hyaluronic acid, aging face, dermal filler, wrinkles, Restylane, Perlane, Juvéderm

\section{Introduction}

The aging phenomenon affects the human face by provoking an array of microscopic and macroscopic complex volumetric changes. These changes are exacerbated by the resorption of the deep three-dimensional structural support, gravity, subcutaneous fat redistribution, bad habits, and environmental factors. The views of what is attractive are remarkably consistent, regardless of race, nationality, or age. The esthetic concepts of beauty demand that certain curves, contours, dimensions, and ratios are fulfilled in order to create harmony and produce an attractive face.

In the past, surgical techniques have dominated the facial rejuvenation field (de Maio 2004). The importance of volume restoration is now recognized, and in many cases has taken precedence over the two-dimensional lifting obtained when using the scalpel. Surgical procedures in some cases may even intensify the apparent loss of volume (Little 2000). Dermal fillers achieve what no other procedure is capable of: they provide volume. Tissue volume augmentation via non-invasive procedures using soft tissue biodegradable fillers can restore the youthful appearance to an aging face by filling out folds and improving fine lines and wrinkles, while proving safe and effective to both male and female patients across all ethnic groups.

Dermal fillers have experienced an astounding demand in the US market since the introduction of bovine collagen-based dermal fillers during the 1980s. The introduction of Restylane ${ }^{\circledR}$, a hyaluronic acid (HA)-based filler at the end of 2003, has increased use of polymer fillers by $700 \%$ (Matarasso et al 2006). Today, HA-based dermal fillers are the fastest non-invasive esthetic procedure in the US, according to the American Society of Aesthetic Plastic Surgeons on February 24, 2006 (Wise and Greco 2006). While the list of injectable fillers continues to expand with the use of different innovative 
compounds, HA fillers still remain the most popular dermal filler agent for the management of facial aging (Lupo 2006). Crosslinked animal non-animal derived HA fillers have been available for more than 18 years in the US and even longer in different countries around the world. These compounds are biocompatible (Fernandez-Cossio and Castaño-Oreja 2006) and have the capacity to retain water at up to 1000 times their volume (Johl and Burgett 2006).

Overall, the recent advances in injection techniques and a better understanding of facial aging can now provide experienced injectors with superior results when using crosslinked hyaluronan dermal fillers for facial remodeling. This article describes the utilization of HA dermal fillers which are approved in the US by the Food and Drug Administration in the management of the aging face.

\section{A brief history of HA}

HA was first discovered by two American scientists at Columbia University in New York City: Karl Meyer who is considered the father of glycosaminoglycan chemistry and his assistant John Palmer (Simoni et al 2002). In 1934 they were able to isolate this substance from bovine vitreous humor and later named it "hyalos" in combination with the uronic sugar also found in the substance. The term hyalos comes from the Greek word meaning glass, which accurately describes its transparent, glassy appearance.

HA was later used commercially for the first time by Endre Balazs who applied for a patent to use this substance as a substitute for egg white in bakery products (Balazs and Denlinger 1989). Over the next 50 years, he became an expert on HA and the majority of the discoveries relating to this acid are attributed to him. During the last two decades, HA has been extensively used in eye surgery, wound repair, and for the treatment of arthritis joints via injection of the knees to aid movement due to its hydration and lubrication properties. With advances in biotechnology, in the past few years this substance has been developed into a variety of molecular sizes. Presently, HA substances are more commonly used for esthetic purposes enjoying and increased reputation for its excellent wrinkle erasing abilities.

\section{Description of HA}

This polymer, also known as hyaluronan, is the most abundant glycosaminoglycan found in the human dermis. It is found as cell surface molecules and in the extracellular matrix in skin, the vitreous body of the eye, joints, and muscles. It is a ubiquitous component of all mammalian connective tissue and responsible for drawing water into the skin giving it volume while binding collagen and elastin fibers into a supportive and protective matrix that gives the skin its structure. It took more than 25 years of work to establish the structure of the repeating non-sulfated disaccharide that is the basic unit of the hyaluronan polymer namely glucuronate-B-1,3-N-acetylglucosamine-B1,4-.

HA is a naturally occurring biopolymer that exhibits no species or tissue specificity. Its structure is identical if it is derived from bacterial cultures, animals, or humans. It is an essential component of the extracellular matrix of all adult animal tissues. Unmodified, natural occurring hyaluronan is rapidly broken down by hyaluronidase and eliminated through the lymphatics and by the hepatic metabolism (Coleman and Grover 2006). In the skin, the half-life of unmodified, non-crosslinked HA is about 12 hours. Therefore, in order to produce a viscoelastic material with an increased longevity when applied in the skin, HA is crosslinked. Currently, there is an array of dermal fillers available to the physician based on crosslinked HA technologies.

\section{HA dermal fillers}

Scientists and physicians are constantly searching for the ideal dermal filler. This ideal filler should be safe and effective, biocompatible, non-immunogenic, easy to distribute and store, and should require no allergy testing. Moreover, it should be low cost, have an acceptable persistency. and be easy to remove if necessary. HA dermal fillers have most of these ideal characteristics (Duranti et al 1998).

HA fillers can easily be removed whenever the practitioner considers necessary by injecting commercially available hyaluronidase (Vitrase ${ }^{\circledR}$; ISTA Pharmaceuticals, CA, USA) into the concerned area (Soparkar et al 2004; Brody 2005; Goldberg and Fiaschetti 2006; Hirsch et al 2007). Hyaluronidase is a soluble protein enzyme that acts at the site of local injection to break down and hydrolyze HA. These important characteristics have made HA-based cosmetic injections the second most popular non-surgical procedure for women and the third most popular procedure for men in 2006 (Winnington 2007).

Several HA fillers are currently commercially available in the US (Table 1) for mid to deep dermal implantation for the correction of moderate to severe facial wrinkles and folds, such as nasolabial folds. Hylaform ${ }^{\circledR}$ was approved in April 2004 (Monheit 2004). This HA filler is composed of HA derived from avian sources and crosslinked with divinyl sulfone (Narins and Bowman 2005). The utilization of Hylaform ${ }^{\circledR}$ dermal filler has substantially diminished since the approval of other HA fillers. Captique ${ }^{\circledR}$ dermal filler is 
Table I FDA-approved hyaluronic acid fillers

\begin{tabular}{ll}
\hline US FDA approved products & Company \\
\hline Hylaform & Genzyme \\
Hylaform Plus & Genzyme \\
Captique & Genzyme \\
Restylane & Medicis Aesthetics \\
Juvederm Ultra & Allergan, Inc \\
Juvederm Ultra Plus & Allergan, Inc \\
Perlane & Medicis \\
Elevess & Anika therapeutics \\
\hline
\end{tabular}

based on non-animal HA and was approved in December 2004. Marketed by Allergan Inc., it will no longer be available after this year (pers comm, D. Moatazedi, Allergan, Inc., Nov 8, 2007).

Presently, the most widely used dermal filler in North America is Restylane ${ }^{\circledR}$. Restylane ${ }^{\circledR}$ was FDA-approved in December of 2003. Since 2003, with the results from the pivotal multicenter, double-blind clinical study, it has been proven that Restylane ${ }^{\circledR}$ is safe and effective in the treatment of nasolabial folds (Narins et al 2003). Perlane ${ }^{\circledR}$, a more viscous version of Restylane $^{\circledR}$, was FDA-approved in 2007. Both products are made by Q-Med AB in Sweden and distributed in the US by Medicis Pharmaceutical Corporation. They are based on "nonanimal stabilized hyaluronic acid" (NASHA) and produced from cultures of Streptococcus equi via a proprietary process crosslinked with 1,4-butanediol diglycidyl ether (BDDE) giving a final concentration of $20 \mathrm{mg} / \mathrm{mL}$. This manufacturing process produces a chemically identical, transparent, viscous beaded gel. Both products are made from the same material and have the same properties, except that Perlane ${ }^{\circledR}$ contains only 8000 HA beads per $\mathrm{mL}$ while Restylane contains 100,000 gel beads.

Restylane ${ }^{\circledR}$ and Perlane ${ }^{\circledR}$ degradation is isovolemic, meaning, it retains most of its initial filler volume throughout the degradation phase. The benefit produced by these fillers is via a volume effect and by attracting and binding water. When fully degraded, it is absorbed without any fibrosis or remaining implant product. Metabolism by-products are water and carbon dioxide. Recent histopathological research with Restylane ${ }^{\circledR}$ has shown that it also stimulates neocollagenesis (Wang et al 2007).

The new HA dermal fillers, Juvéderm ${ }^{\mathrm{TM}}$ Ultra and Juvéderm ${ }^{\mathrm{TM}}$ Ultra Plus injectable gels, are distributed by Allergan, Inc. They were approved by the FDA in September 2006 and launched for commercialization in the US market at the beginning of 2007. Both products feature a novel crosslinking process called Hylacross which provides a concentration of $24 \mathrm{mg} / \mathrm{mL}$ of HA. Juvéderm ${ }^{\mathrm{TM}}$ Ultra Plus is a more robust formulation with a higher crosslinked composition of $8 \%$ versus $6 \%$ in the Juvéderm ${ }^{\mathrm{TM}}$ Ultra. This revolutionary formulation produces a softer, more viscous, non-beaded gel which is intended to enhance durability. A prospective double-blind, randomized, within-subject controlled, multi-center clinical trial comparing Juvéderm ${ }^{\mathrm{TM}}$ Ultra or Juvéderm ${ }^{\mathrm{TM}}$ Ultra Plus to bovine collagen have shown an increased persistence for the HA products (Package Insert Juvéderm Ultra L040-04 12/06; Juvéderm Ultra Plus L041-04 12/06). Throughout the 24-week study period, Juvéderm ${ }^{\mathrm{TM}}$ Ultra and Juvéderm $^{\mathrm{TM}}$ Ultra Plus injectable gel provided a clinically and statistically significant improvement in nasolabial severity. Based on new clinical data demonstrating that the effects with a single treatment of either formulations may last for up to 12 months, the FDA have granted a label extension for Juvéderm ${ }^{\mathrm{TM}}$ Ultra and Juvéderm ${ }^{\mathrm{TM}}$ Ultra Plus in June, 2007 (Allergan, Inc. 2007).

Elevess $^{\mathrm{TM}}$ is the latest HA approved by the FDA, in July 2007. The product, manufactured by Anika Therapeutics, MA, USA, is based on chemically modified non-animal HA proprietary technology which incorporates $0.3 \%$ lidocaine hydrochloride as a component of the treatment syringe. The concentration of HA in this product is the highest available at $28 \mathrm{mg} / \mathrm{mL}$. Elevess ${ }^{\mathrm{TM}}$ crosslinker is p-phenylene bisetheyl carbodimide (BCDI). At time of publication, this product is not commercially available.

All of these HA fillers available in the US are approved for the cosmetic improvement of the nasolabial fold; however, used off-label, injectable HA dermal fillers are useful for restoring volume to localized areas such as the cheeks, as well as reduction of the oral commissures, marionette lines, forehead lines, temple areas, tear trough, jowls, and lips.

The HA dermal fillers on the horizon are Puragen, Puragen Plus, Prevelle, Prevelle Plus, Belotero, and Teosyal family of products (Table 2). Puragen and Puragen plus are based on double crosslinked (DXL ${ }^{\mathrm{TM}}$ ) technology with non-animal HA chains. DXL ${ }^{\mathrm{TM}}$ technology increases the resistance to degradation once the product is implanted. Puragen Plus product will incorporate lidocaine for pain management. Prevelle and Prevelle Plus will be less robust formulationa and according to the manufacturer will produce

Table 2 Hyaluronic acid dermal fillers to come and not yet available in the US

\begin{tabular}{ll}
\hline Product & Company \\
\hline Teosyal & Teoxane, Switzerland \\
Puragen Plus & Mentor, CA, USA \\
Prevelle & Mentor, CA, USA \\
Belotero & Merz pharmaceuticals, NC, USA \\
\hline
\end{tabular}


less immediate post-injection adverse events. These four products are manufactured by Mentor Corporation, CA, USA. Belotero, manufactured by Anteis SA, Geneva, Switzerland and distributed by Merz Pharmaceutical LLC, is also based on double crosslinked technology called Cohesive Polydensified Matrix (CPM) with BDDE and nonanimal HA chains. Teosyal family of products consists of 7 formulations based on monophasic, non-animal HA, crosslinked with BDDE.

Having an expanded armamentum physicians have now several choices of HA dermal fillers. It is important to keep in mind that not all HA fillers are created equal. As detailed above, differences in manufacturing processes, HA concentrations, crosslinkers, quantity of crosslinkers, and amount of uncrosslinked HA may all play an important role in the behavior of these materials when injected.

There are several techniques that can be utilized for the facial implantation of HA dermal fillers. Linear threading, serial puncture, fanning, and cross-hatching, or a combination of all four has been successfully used for the management of the aging face with HA dermal fillers. As with all injectable procedures, the patient desires and expectations should be carefully reviewed (Kelly 2007). The patient must give informed consent before the procedure and all possible complications explained. The patient's skin must be cleaned and conditioned with the application of numbing topical creams such as LMX4 (4\% lidocaine) (Biopelle, MI, USA) or the new Pliaglis ${ }^{\mathrm{TM}}$ ( $7 \%$ lidocaine and $7 \%$ tetracaine) cream that forms a pliable peel on the skin when exposed to air (Galderma Pharma SA, TX, USA) for at least 30 minutes. Some practitioners prefer to use regional nerve blocks (Rao et al 2005; Matarasso et al 2006).

Once the HA filler is injected, it might be lightly massaged to conform to the contour of the surrounding tissues. It is important to remember that these fillers are hygroscopic and may increase correction up to $15 \%$ after injection (Monheit 2006). It is good clinical practice to get an understanding of the patient's realistic goals and evaluate whether or not HA fillers can fulfill their desires during consultation. Conceptually, the aging face can be divided into upper, middle, and lower thirds. Using this approach, we will discuss the clinical strategies for addressing each of these facial regions.

\section{Upper face (Table 3) Glabellar lines}

The symmetrical lines between the eyebrows that develop by the prolonged muscular action of the corrugator supercilii and procerus muscles respond extremely well to botulinum
Table 3 Preferred hyaluronic acid products for the upper face

\begin{tabular}{ll} 
Area & \\
\hline Glabellar & Restylane \\
Forehead & Restylane \\
Periocular & Perlane and/or Restylane \\
\hline
\end{tabular}

toxin therapy (Carruthers and Carruthers 2003). However, in cases where the patient has deep glabellar furrows, HA fillers can be used depending on the severity of the defect. Botulinum toxins and dermal fillers combined produce the best results for the treatment of this area. It is important that implantation of the HA filler is completed without overcorrection. Moreover, this area is very sensitive to occlusion of blood vessels; therefore, fillers must be applied superficially to minimize the risk of cutaneous necrosis.

\section{Forehead lines}

Forehead horizontal lines are the resultant defect produced by the dynamic action of the frontalis muscles (Stennert 1994). Wrinkles in this area could be dynamic or non-dynamic. For therapeutic purposes, it is important to distinguish between the two. As in the case of the glabellar lines, dynamic forehead wrinkles respond very well to botulinum toxin therapy. Non-dynamic forehead wrinkles result mostly from the volume loss of the underlying tissue. Injected superficially via serial puncture technique without overcorrection, HA fillers can provide good esthetic results.

\section{Periocular areas}

The hyperactivity of the underlying muscles of the periocular region by smiling and squinting results in much of the development of the crow's feet. Most of these wrinkles respond best to botulinum toxin therapy. Deeper periocular wrinkles qualify and respond well to treatment with HA dermal fillers using retrograde linear threading or serial puncture technique via a 32-gauge needle. The delicate nature of the skin in this area is the primary concern. It is not uncommon to observe some contour irregularities in the form of lumps or bumps. In addition, due to the rich subdermal vascular plexus, this area is very prone to significant bruising.

Facial aging affecting fat atrophy of the temple area responds well to HA treatment. Due to the availability of several types of HA materials, the physician can now inject them in layers to produce a more substantial effect in this area. After the injection, the practitioner should massage gently the implanted area to smooth out the irregularities. Ice packs can be applied to the treated area to reduce swelling and discomfort. 
The facial aging process results in dropping and receding brows. The injection of HA fillers has become very popular to elevate the brow and produce a younger facial expression. Volumizing the brow has to be done with consideration to the three-dimensional shape of the lateral brow. The end result is a desirable arching contour. The filler material is injected just below the cilia and implanted using the anterograde technique as the needle is pushed forward across the brow to the notch. Due to the malleable properties of these fillers, the material can be contoured by pressing it along the brow between the thumb and the index fingers. It is our experience that Restylane ${ }^{\circledR}$ injected in this area might last more than 9 months. Some physicians prefer to inject Restylane ${ }^{\circledR}$ deeper at the level of the periosteum. Longer tenderness in the treated area has been reported when this technique is use with this material (Matarasso et al 2006). Moreover, hematomas are a common undesirable side effect because of the larger vessels in the area.

\section{Midface (Table 4)}

Facial aging consistently produces increasing prominence of the midface, hollow eyes, nasolabial folds, cheeks, lips, jowls, and chin.

\section{Inferior orbital rim}

Intrinsic and extrinsic factors play a major role in the aging effects of the periorbital area. Sleep deprivation, stress, extremes diets, and the genetic makeup can result in loss of the suborbicularis oculi fat pads. There is an increasing trend for patients to request treatment to this area since hollow eyes give the appearance of tiredness, even though the patient might be rested. HA fillers do provide a refreshed facial expression when properly injected. Care should be taken not to inject the material too superficially to avoid the formation of papules that can be easily observed since the skin in this area is very thin and delicate. HA is injected into the deep cutaneous tissues using the serial puncture technique or threading technique, injecting the product as the needle is withdrawn (Kane 2007). Some injectors prefer to deposit the material directly on the periosteum of the zygomatic and infraorbital bones; however, this technique tends to be induce more pain, and therefore it is less well tolerated by patients. Others prefer to deposit the filler intraorally. Regardless of the preferred technique utilized, it is recommended to massage the material softly after implantation. Due to the high vascularity present in this area, bruising is a major side effect when fillers are injected into the inferior orbital rim. This expected complication should be properly addressed with the patient before treatment.
Table 4 Preferred hyaluronic acid products for the midface

\begin{tabular}{ll}
\hline Area & \\
\hline Cheeks & Perlane \\
Nasolabial folds & Juvéderm Ultra and/or Restylane \\
\hline
\end{tabular}

\section{Cheeks and nasolabial folds}

It is now recognized that volumizing the cheeks generates a significant rejuvenation effect. With the passage of time, the facial triangle of a young face where the apex is at the chin gets reversed, and the volume that was once on the midface moves down reversing the orientation of this triangle of youth. Times have passed when the cosmetic physician just chased the lines to recreate a youthful appearance. Presently, the cheek and malar areas are rejuvenated by injecting HA dermal fillers, especially the most robust formulations such as Perlane ${ }^{\circledR}$ or Juvéderm ${ }^{\mathrm{TM}}$ Ultra Plus via serial puncture or linear threading techniques in the lower dermis as the needle is withdrawn while full correction is attempted. Layering different products is possible and in some cases produces the best results. By volumizing the cheek and the lost malar fat pads, not only is the nasolabial fold corrected but, moreover, an overall facial rejuvenation is achieved. The face looks younger and plumped. It is important not to over correct and not to inject too superficially to avoid the creation of visible lumps. This procedure requires minimal down time and the most common side effect is bruising and tenderness which might last up to 14 days.

\section{Lower face (Table 5)}

Perioral area

Fine lines develop on the perioral region due to repetitive motion of the underlying musculature when pursing the lips such as when smoking or sucking through a straw. These fine lines can be treated by injecting HA fillers with a 33-gauge needle. This techniques allows for breaking up the HA gel, especially in the case of Restylane ${ }^{\circledR}$ into a finer material more suitable for the treatment of these fine lines without overcorrection.

Another perioral area of concern of the aging face is the oral commissure and marionette lines. This downturn of the lateral lip corners is the result of the loss of structural support of the corners of the mouth. These changes result in the appearance of sadness and old age. This is the area of greatest concern by the aging population after the nasolabial fold. The oral commissures and marionette lines can be treated safely and effectively with a combination of HA fillers by using the serial puncture and/or 
Table 5 Preferred hyaluronic acid products for the lower face

\begin{tabular}{ll}
\hline Area & \\
\hline Perioral & Restylane \\
Lips & Juvederm Ultra \\
Crease of chin & Juvederm Ultra \\
Jawline & Perlane \\
\hline
\end{tabular}

cross-hatching techniques while elevating the affected area with the non-injecting hand before implantation of the material. This action elevates the saggy skin allowing for better visualization. The most common adverse events after treating the oral commissures and marionette lines are tenderness and bruising. A more serious side effect is tissue necrosis. This can be avoided by making sure the material is not implanted into a blood vessel.

\section{Lips}

One of the most requested areas for tissue augmentation in young and old patients alike are the lips. Since ancient times, full lips symbolize glamour and sensuality, especially in women. Lips are a key esthetic feature of the lower face. Lips are also affected by the aging process. Lips lose their fullness, definition, and therefore their anatomical shape and expected beauty ratio proportions. They become narrow, pale, and flat. HAs are ideal fillers to replenish the volume lost and re-establish the pleasing esthetic look of well proportioned and voluminous lips. It is important to consult the patient carefully to find out if she/he wants to restore their lips to their former appearance, or if they want a different, more sumptuous look (Bousquet and Agerup 1999).

Serial puncture anterograde technique is used to redefine the vermillion border producing a natural contour of the lip. For replenishing the volume of the mucosa of the lip, the robust HA filler formulations can be utilized using retrograde serial puncture techniques. The most robust formulations provide for longer lasting results (Brandt and Cazzaniga 2007).

\section{Jawline}

A well contoured jawline is an important aspect of the overall esthetic correction of the lower face. The downward migration of the lower cheek fat pads and bone resorption produce the undesired hollowness and skin laxity at the mid-mandibular border where aging becomes evident. HA fillers are excellent materials to reshape and contour the atrophied jowls by lifting and tightening the senescent sagging cutaneous tissues to create a younger-looking appearance.
HA dermal fillers are injected in this area by serial puncture and linear threading techniques. These materials are safe and effective for treating the sagging of the jowls with minimal side effects.

\section{Crease of chin}

Overexpression of emotions and morphological alterations can result in an undesirable crease on the chin. Because the loss of dermal collagen and subcutaneous fat pads add to this insult, patients find this defect very unattractive. HA dermal fillers provide a successful and lasting treatment effect to this area. Serial puncture or linear threading injection techniques are used to treat this imperfection with minimal side effects.

\section{Adverse events}

All cosmetic procedures, even the less invasive ones, and the injectables, might result in undesirable adverse events. Therefore, HA-based dermal fillers are not devoid of inducing complications. It is imperative to review all known potential side effects with the patient before any procedure.

The most common localized side effects encountered after treatments with HA dermal fillers are temporary pain, induration, bruising, tenderness, itching, edema, and erythema at the injection site (Lowe et al 2005). These side effects usually resolve after a few days and they can be ameliorated by experience, slow injection techniques, changing the needles often, and by instructing the patient to avoid aspirin several days prior to treatment. There have been reports of hypersensitivity reactions ranging from $0.0005 \%$ to $0.42 \%$ (Brown and Frank 2003; Hönig et al 2003) and, in rare cases, necrosis or embolization might occur (Schanz et al 2002; Hirsch et al 2007). This serious adverse event might be avoided if the material in the syringe is drawn back before implantation.

\section{Conclusion}

Esthetic facial volume restoration along with all other components of the aging face must be adequately addressed to successfully rejuvenate the face. The safe and effective track record of HA dermal fillers makes them currently the most popular filling material. This popularity will continue to increase as the aging population searches for viable options to correct the signs of aging without surgery. The utilization of these fillers by trained professionals provides an effective and safe therapy for the management of the aging face. 


\section{References}

Allergan Inc. News Release 2007. Accessed 26 June 2007. URL: http://www. fiercebiotech.com

Balazs EA, Denlinger JL. 1989. Clinical uses of Hyaluronan. Ciba Found Symp, 143:265.

Bousquet MT, Agerup B. 1999. Restylane lip implantation: European experience. Operative Techniques in Oculoplastic, Orbital, and Reconstructive Surgery, 2:172-6.

Brandt F, Cazzaniga A. 2007. Hyaluronic acid fillers: Restylane and Perlane. In Maas CS (Guest Ed). Facial Plastic Surgery Clinics of North America. Philadelphia: Elsevier Saunders, pp. 63-76.

Brody H. 2005. Use of hyaluronidase in the treatment of granulomatous hyaluronic acid reactions or unwanted hyaluronic acid misplacement. Dermatol Surg, 31:893-7.

Brown LH, Frank PJ. 2003. What's new in fillers? J Drugs Dermatol, 2:250-3.

Carruthers J, Carruthers A. 2003. A prospective, randomized, parallel group study analyzing the effect of BTX-A (Botox) and nonanimal sourced hyaluronic acid (NASHA, Restylane) alone in combination compared with NASHA (Restylane) alone in severe glabellar rhytides in adult female subjects: treatment of severe glabellar rhytides with a hyaluronic acid derivative compared with the derivative and BTX-A, Dermatol Surg, 29:802-9.

Carruthers J, Klein AW, Carruthers A, et al. 2005. Safety and efficacy of Restylane for improvement of mouth corners. Dermatol Surg, $31: 1-5$.

Coleman KR, Carruthers J. 2006. Combination therapy with Botox and fillers: the new rejuvenation paradigm. Dermatol Ther, 16:177-88.

Coleman SR, Grover R. 2006. The anatomy of the aging face: volume loss and changes in 3-dimensional topography. 2006. Aesthetic Surgery Journal, Jan/Feb s4-s9.

De Maio M. 2004. the minimal approach: an innovation on facial cosmetic procedure. Aesthetic Plast Surg, 28:295-300.

Duranti F, Salti G, Bovani B, et al. 1998. Injectable hyaluronic acid gel for soft tissue augmentation, a clinical and histological study. Dermatol Surg, 24:1317-25.

Fernández-Cossío S, Castaño-Oreja MT. 2006. Biocompatibiliy of two novel dermal fillers: histological evaluation of implants of a hyaluronic acid filler and a polyacrylamide filler. Plast Reconstr Surg, 117:1789-96.

Golberg RA, Fiaschetti D. 2006. Filling the periorbital hollows with hyaluronic acid gel: initial experience with 244 injections. Ophthal Plast Reconstr Surg, 22:335-41.

Hirsch RJ, Lupo M, Cohen JL, Duffy D. 2007. Case Reports: delayed presentation of impending necrosis following soft tissue augmentation with hyaluronic acid and successful management with hyaluronidase. J Drugs Dermatol, 6:325-8.

Hönig JF, Brink U, Korabiowska M. 2003. Severe granulomatous allergic tissue reaction after hyaluronic acid injection in the treatment of facial lines and its surgical correction. J Craniof Surg, 14:197-200.
Johl SS, Burgett RA. 2006. Dermal filler agents: a practical review. Curr Opin Ophthalmol, 14:471-9.

Kane MAC. 2007. Advanced techniques for using Restylane in the lower eyelids. Aesthetic Surg, 27:90-2.

Kelly PE. 2007. Injectable success: from fillers to Botox. Facial Plast Surg, 23:7-18.

Little JW. 2000. Volumetric perceptions in midfacial aging with altered priorities for rejuvenation. Plast Reconstr Surg, 105:252-66.

Lowe NJ, Maxwell CA, Patanaik R. 2005. Adverse reactions to dermal fillers: review. Dermatol Surg, 31:1616-25.

Lupo MP. 2006. Hyaluronic acid fillers in facial rejuvenation. Semin Cutan Med Surg, 25:122-6.

Matarasso SL, Carruthers JD, Jewell ML and the Restylane Consensus Group. 2006. Consensus recommendations for soft tissue augmentation with nonanimal stabilized hyaluronic acid (Restylane). Plast Reconstr Surg, 117:3S-34S.

Monheit GD. 2004. Hylaform: a new hyaluronic acid filler in soft tissue augmentation. Facial Plast Surg, 20:153-7.

Monheit GD, Coleman KM. 2006. Hyaluronic acid fillers. Dermatol Ther, $16: 141-50$.

Narins RS, Bowman PH. 2005. Injectable skin fillers. Clin Plast Surg, 32:151-62.

Narins RS, Brandt FS, Leyden J, et al. 2003. A randomized, double-blind, multicenter comparison of the efficacy and tolerability of Restylane versus Zyplast for the correction of nasolabial folds. Dermatol Surg, 29:588-95.

Rao J, Chi GC, Goldman MP. 2005. Clinical comparison between two hyaluronic acid-derived fillers in the treatment of nasolabial folds: Hylaform versus Restylane. Dermatol Surg, 31:1587-90.

Schanz S, Schippert W, Ulmer A, et al. 2002. Arterial embolization caused by injection of hyaluronic acid (Restylane). Br J Dermatol, 146:928-9.

Simoni RD, Hill RL, Vaughan M, et al. 2002. JBC Centennial. The discovery of hyaluronan by Karl Meyer. J Biol Chem, 277:61-2.

Soparkar CN, Patrinely JR, Tschen J. 2004. Erasing Restylane. Ophthal Plast Reconstr Surg, 20:317-8.

Stennert E. 1994. Why does the frontalis muscle "never" come back? Functional organization of the mimic musculature. Eur Arch Otorhinolaryngol, S91-5.

Wang F, Garza L, Kang S, el al. 2007. In vivo stimulation of De Novo collagen production caused by cross-linked hyaluronic acid dermal filler injections in photodamaged human skin. Arch Dermatol, 143:155-63.

Winnington P. 2007. Tuning-In to the demand for dermal fillers. Practical Dermatology, 36-9.

Wise JB, Greco T. 2006. Injectable treatments for the aging face. Facial Plast Surg, 22:140-6. 
